\title{
On the bootstrap current in stellarators and tokamaks
}

\author{
P. Helander, J. Geiger and H. Maaßberg
}

Max-Planck-Institut für Plasmaphysik, 17491 Greifswald, Germany

The expression for the long-mean-free-path limit of the bootstrap current in stellarators is rederived in such a way that the expansion procedure is identical to that used in the corresponding calculation for a tokamak. In addition, the first correction due to finite collisionality is calculated and shown to vanish in quasi-isodynamic configurations without net current. This correction, which is proportional to the square root of the collisionality, is found to compare well with a numerical solution of the first-order drift kinetic equation in spherical tokamak geometry. Numerically, it appears that there is a similar correction in general stellarator geometry, which however depends on the value of the radial electric field.

PACS numbers. 52.55.Hc, 52.25.Fi 


\section{Introduction}

The bootstrap current was predicted and calculated for tokamak geometry four decades ago by Galeev [1] and Bickerton, Connor and Taylor [2]. The latter also patented it but the patent has long expired. Experimentally, it was discovered only in the mid 1980's [3] but has since then proven to be of great importance to both tokamaks and stellarators. In tokamaks, it can contribute significantly to the total plasma current and thereby to confinement, whilst the bootstrap current in stellarators can either increase or reduce the rotational transform $\iota$. In devices with small magnetic shear, it can significantly affect the radial $\iota$-profile and introduce unwanted low-order rational surfaces into the plasma. It can also interfere with island divertor operation.

A proper calculation of the bootstrap current in stellarator geometry was first done by Shaing and Callen [4], using the Hirshman-Sigmar moment method, which was followed by a more direct calcuation by Boozer and Gardner [5]. Both these derviations are complicated, and it is not entirely clear whether they actually give the same result. Therefore, a more direct calculation is presented in Secs. II-IV below, which arrives at the same result as Shaing and Callen but without using the moment formalism. In addition, the present calculation has the merit of being identical in tokamaks and stellarators, since it does not rely on an expansion characteristic of the $1 / \nu$-regime of collisionality peculiar to stellarators and used in Refs. [6, 7]. In Sec. V, the first correction due to finite collisionality is discussed. This correction, which was first calculated by Hinton and Rosenbluth, is proportional to the square root of the collision frequency and tends therefore to be important even in fairly low-collisionality plasmas. The Hinton-Rosenbluth calculation was performed for the case of a large-aspect-ratio tokamak with circular flux surfaces and is here generalized to arbitrary axisymmmetric geometry. As shown in Sec V.B, it is then also relevant to quasi-isodynamic stellarators. We also compare the analytical result with a numerical solution of the drift kinetic equation in Sec. VI and find excellent agreement in the appropriate collisionality range. In the penultimate section, we finally briefly discuss the collisionality dependence of the bootstrap current in a classical stellarator. 


\section{Adjoint equation}

The first-order drift kinetic equation for each species $a$ is

$$
v_{\|} \nabla_{\|} f_{a 1}-C_{a}\left(f_{a 1}\right)=-\mathbf{v}_{d a} \cdot \nabla f_{a 0}-\frac{e_{a} v_{\|} \nabla_{\|} \phi_{1}}{T_{a}} f_{a 0},
$$

where we have neglected any effect of the radial electric field arising through a term $\mathbf{v}_{E} \cdot \nabla f_{a 1}$, which is often kept in neoclassical stellarator calculations. The gradient is taken holding the velocity $v$ and the normalized magnetic moment $\lambda=v_{\perp}^{2} / v^{2} B$ fixed, so that the mirror force is contained in the first term in Eq. (1). The bootstrap current is found from the odd part (in $v_{\|}$) of the distribution function,

$$
J_{\|}=\sum_{a} J_{a \|}=\sum_{a} e_{a} \int v_{\|} f_{a 1} d^{3} v
$$

The variation of the electrostatic potential $\phi_{1}$ within the magnetic surface is readily eliminated by writing

$$
f_{a 1}=h_{a}-\frac{e_{a} \phi_{1}}{T_{a}} f_{a 0},
$$

so that the equation for $h_{a}$ is the same as that for $f_{a 0}$ but without the last term in Eq. (1). For a pure plasma with large electron-ion mass ratio, we obtain

$$
\begin{gathered}
v_{\|} \nabla h_{i}-C_{i i}\left(h_{i}\right)=-\mathbf{v}_{d i} \cdot \nabla f_{i 0} \equiv S_{i} \\
v_{\|} \nabla_{\|} h_{e}-C_{e 0}\left(h_{e}\right)=-\mathbf{v}_{d e} \cdot \nabla f_{e 0}+\nu_{D}^{e i} \frac{m_{e} v_{\|} V_{i \|}}{T_{e}} f_{e 0} \equiv S_{e}
\end{gathered}
$$

where

$$
C_{e 0}=C_{e e}+\nu_{D}^{e i}(v) \mathcal{L},
$$

with $\nu_{D}$ the pitch-angle deflection frequency [8] and

$$
\mathcal{L}=\frac{2 v_{\|}}{v^{2} B} \frac{\partial}{\partial \lambda} \lambda v_{\|} \frac{\partial}{\partial \lambda}
$$

the pitch-angle scattering operator.

Instead of solving these equations directly, we shall use the method of Antonsen and Chu [9] and instead solve the adjoint equation

$$
v_{\|} \nabla_{\|} g_{a}+C_{a 0}\left(g_{a}\right)=-\frac{v_{\|} B}{\left\langle B^{2}\right\rangle} f_{a 0},
$$

where $\left\langle B^{2}\right\rangle$ is the flux-surface average (defined below) of $B^{2}$ and $C_{a 0}$ denotes either $C_{i i}$ or $C_{e 0}$. Both these operators are self-adjoint in the sense that

$$
\int \frac{k_{1}}{f_{a 0}} C_{a 0}\left(k_{2}\right) d^{3} v=\int \frac{k_{2}}{f_{a 0}} C_{a 0}\left(k_{1}\right) d^{3} v
$$


for any functions $k_{1}$ and $k_{2}$. With the help of this property and the solution of Eq. (4), the current density of each species can be calculated by

$$
\left\langle J_{a \|} B\right\rangle=\left\langle e_{a} B \int h_{a} v_{\|} d^{3} v\right\rangle=e_{a}\left\langle B^{2}\right\rangle\left\langle\int \frac{g_{a} S_{a}}{f_{a 0}} d^{3} v\right\rangle
$$

where $S_{a}$ denotes the right-hand side of Eqs. (2) and (3), respectively. The fluxsurface average is defined as the volume average between two neighboring flux surfaces. Explicitly, in magnetic coordinates $(\psi, \theta, \varphi)$, where $\theta$ and $\varphi$ are poloidal and toroidal angles, $\psi$ the toroidal flux and $\mathbf{B}=\nabla \psi \times \nabla \theta+\iota \nabla \varphi \times \nabla \psi$, the average is defined as

$$
\langle\cdots\rangle=\int_{0}^{2 \pi} d \theta \int_{0}^{2 \pi}(\cdots) \sqrt{g} d \varphi / \int_{0}^{2 \pi} d \theta \int_{0}^{2 \pi} \sqrt{g} d \varphi,
$$

with $\sqrt{g}=1 /[\nabla \psi \cdot(\nabla \theta \times \nabla \varphi)]$.

\section{Solving the kinetic equation}

To solve Eq. (4) when the collisionality is low, i.e., when the bounce frequency exceeds the collision frequency, we expand

$$
g_{a}=g_{a 0}+g_{a 1}+\cdots
$$

in the same way as when treating the banana regime in a tokamak,

$$
\begin{aligned}
v_{\|} \nabla_{\|} g_{a 0} & =0 \\
v_{\|} \nabla_{\|} g_{a 1}+C_{a 0}\left(g_{a 0}\right) & =-\frac{v_{\|} B}{\left\langle B^{2}\right\rangle} f_{a 0} .
\end{aligned}
$$

The function $g_{a 0}(v, \lambda, \sigma, \psi)$, where $\sigma=v_{\|} /\left|v_{\|}\right|$, thus depends only on constants of the lowest-order guiding centre motion (without drifts). On a flux surface with irrational rotational transform, $\iota, g_{a 0}$ is thus a flux function, and because of continuity this must also be the case on rational surfaces. Incidentally, this argument suggests that the long-mean-free-path limit we are considering is approached much more slowly in a stellarator than in a tokamak as the collision frequency is decreased. In the tokamak, it is sufficient that the mean free path should exceed a few times the connection length; in the stellarator, it must be long enough that a particle covers much of the flux surface between collisions. As we shall see numerically in the penultimate section, the longmean-free-path limit is indeed only appropriate at extremely low collision frequencies. 
As usual in neoclassical theory, the function $g_{a 0}$ is determined by the solvability constraint following from Eq. (8). Since the right-hand side of this equation is odd in $v_{\|}, g_{a 0}$ vanishes in the trapped region of velocity space [8]. In the passing region, the integrability constraint that follows from Eq. (8) by multiplying by $B / v_{\|}$and taking the flux surface average,

$$
\left\langle\frac{B C_{a 0}\left(g_{a 0}\right)}{v_{\|}}\right\rangle=-f_{a 0},
$$

determines $g_{a 0}$.

This equation can only be solved analytically (or even numerically, so far) if the collision operator is simplified and replaced by an operator of the form

$$
C_{a 0}\left(g_{a}\right)=\nu_{D}^{a} \mathcal{L}\left(g_{a}\right)+M(v, \psi, \theta, \varphi) v_{\|} f_{a 0} .
$$

Historically, the rationale for approximating the collision operator by pitch-angle scattering (the first term) was that Rosenbluth, Hazeltine and Hinton [10] proved that this operator is sufficiently accurate when the fraction of trapped particles is small, provided that a momentum-restoring term (here proportional to $M$ ) is also introduced to ensure parallel momentum conservation. The constant $M$ can then be taken to be independent of velocity, but it has later emerged that allowing $M$ to depend on $v$ is necessary for obtaining results that are reasonably accurate when the trapped-particle fraction is finite $[8,11,12]$. For our purposes it is important that the calculation can be divided into two steps: first the kinetic equation (4) is solved without the momentumrestoring term, and in a second step the solution to the equation with $M \neq 0$ can be found without solving another differential equation [13, 14]. This circumstance enables us to focus on the simpler case with $M=0$, where Eq. (9) has the well-known solution $[8,10]$

$$
g_{a 0}=\frac{\sigma v f_{a 0}}{2 \nu_{D}^{a}} \int_{\lambda}^{1 / B_{\max }} \frac{d \lambda^{\prime}}{\left\langle\sqrt{1-\lambda^{\prime} B}\right\rangle} .
$$

In addtion, we may ignore the term involving $V_{i \|}$ in $S_{e}$, see Eq. (3), since it is of the same form as the momentum-restoring term in the collision operator and can therefore also be accounted for separately. 


\section{Bootstrap current}

In the expression (5) for the current, we note that $g_{a 0}$ does not contribute since it is odd in $v_{\|}$. We therefore need

$$
g_{a 1}=\int_{l_{\max }}^{l} f_{a 0}\left[-\frac{B}{\left\langle B^{2}\right\rangle}+\frac{1}{B} \frac{\partial}{\partial \lambda}\left(H\left(B_{\max }^{-1}-\lambda\right) \frac{\lambda \xi}{\langle\xi\rangle}\right)\right] d l^{\prime}
$$

from Eqs. (8) and (11), where $\xi=\sqrt{1-\lambda B}$. The Heaviside function $H$ appearing in this expression vanishes in the trapped domain, and we have fixed an arbitrary integration constant by taking the integral from the point $l_{\max }$ where the field strength is the largest, $B\left(l_{\max }\right)=B_{\max }$. Substituting this expression in Eq. (5) gives the current as a sum of two terms,

$$
\left\langle J_{a \|} B\right\rangle=J_{a 1}+J_{a 2},
$$

corresponding to the two terms in the integrand of Eq. (12). The first one is

$$
J_{a 1}=\left\langle e_{a} B \int \frac{\partial f_{a 0}}{\partial r} 2 \pi v^{2} d v \int_{0}^{1 / B} \frac{\mathbf{v}_{d a} \cdot \nabla r}{\xi} d \lambda \int_{l_{\max }}^{l} B d l^{\prime}\right\rangle,
$$

where $r$ is an arbitrary flux-surface label and

$$
\mathbf{v}_{d a} \cdot \nabla r=\frac{m_{a} v^{2}}{e_{a}} \xi(\mathbf{b} \times \nabla r) \cdot \nabla\left(\frac{\xi}{B}\right) .
$$

It is straightforward to carry out the integrals over $v$ and $\lambda$ to obtain

$$
J_{a 1}=p_{a} A_{1 a}\left\langle(\mathbf{B} \times \nabla r) \cdot \nabla\left(\frac{1}{B^{2}}\right) \int_{l_{\max }}^{l} B d l^{\prime}\right\rangle,
$$

where we have written

$$
A_{1 a}=\frac{d \ln p_{a}}{d r}+\frac{e_{a}}{T_{a}} \frac{d \Phi}{d r},
$$

with $\Phi(r)$ the equilibrium electrostatic potential. If, following Ref. [15], we define

$$
g_{2}(l)=B^{2} \int_{l_{\max }}^{l}(\mathbf{b} \times \nabla r) \cdot \nabla B^{-2} d l^{\prime}
$$

we obtain

$$
J_{a 1}=-p_{a} A_{1 a}\left\langle g_{2}\right\rangle
$$

The second term in Eq. (12) makes the following contribution to the current,

$$
J_{a 2}=-\left\langle B^{2}\right\rangle\left\langle e_{a} \int \frac{\partial f_{a 0}}{\partial r} 2 \pi v^{2} d v \int_{0}^{1 / B_{\max }} \frac{B \mathbf{v}_{d a} \cdot \nabla r}{\xi} d \lambda \int_{l_{\max }}^{l} \frac{\partial}{\partial \lambda}\left(\frac{\lambda \xi}{\langle\xi\rangle}\right) \frac{d l^{\prime}}{B}\right\rangle,
$$


where we substitute the drift velocity (13) and integrate by parts in $\lambda$ to obtain

$$
J_{a 2}=\frac{3 p_{a} A_{1 a}\left\langle B^{2}\right\rangle}{4} \int_{0}^{1 / B_{\max }} \frac{\left\langle g_{4}\right\rangle}{\langle\xi\rangle} \lambda d \lambda
$$

with

$$
g_{4}(\lambda, l)=\xi \int_{l_{\max }}^{l}(\mathbf{b} \times \nabla r) \cdot \nabla \xi^{-1} d l^{\prime}, \quad\left(\lambda<1 / B_{\max }\right) .
$$

The total current of each species is thus

$$
\left\langle J_{a \|} B\right\rangle=J_{a 1}+J_{a 2}=p_{a} A_{1 a}\left(\frac{3\left\langle B^{2}\right\rangle}{4} \int_{0}^{1 / B_{\max }} \frac{\left\langle g_{4}\right\rangle \lambda d \lambda}{\langle\sqrt{1-\lambda B}\rangle}-\left\langle g_{2}\right\rangle\right),
$$

as originally found by Shaing and Callen [4] using a very different argument.

As already mentioned, the second term in the collision operator (10) has been neglected but can be accounted for separately without solving another differential equation. This is accomplished by the moment method of Hirshman and Sigmar $[8,16]$ employed in Ref. [4] and, to higher accuracy, by the methods described in Refs. [13, 14]. We will not go into the details here, but remark that the resulting bootstrap current then contains terms that are proportional to the pressure and temperature gradients of all the particle species in the plasma.

\section{$5 \quad$ Finite collisionality}

The result (14) is valid in the collisionless limit. Hinton and Rosenbluth [17] considered the effect of a small but finite collisionality in tokamak geometry and showed that the first correction to the bootstrap current is proportional to the square root of the collisionality and therefore cannot be obtained by simply continuing the expansion (6) to higher order. Instead, it arises from a boundary-layer analysis of the region around the trapped-passing boundary, $\lambda=1 / B_{\max }$. Because $g_{a 0}$ vanishes in the trapped region and is equal to Eq. (11) in the passing region, its derivative $\partial g_{a 0} / \partial \lambda$ is discontinuous at the boundary, making the collision operator infinte, since the pitch-angle scattering operator contains two $\lambda$-derivatives. Collisions therefore need to be retained in a boundary layer whose width is proportional to the square root of the collisionality. In this layer, Eq. (7) needs to be replaced by

$$
v_{\|} \nabla_{\|} g_{a}+C_{a 0}\left(g_{a}\right)=0
$$


with boundary conditions obtained by asymptotic matching to the collisionless solution $g_{a 0}$ away from $\lambda=1 / B_{\max }$.

\section{A. Tokamak with arbitrary cross section}

Alternatively, one can of course perform a similar calculation for the original kinetic equation

$$
v_{\|} \nabla_{\|} f_{a 1}-C_{a}\left(f_{a 1}\right)=-\mathbf{v}_{d a} \cdot \nabla f_{a 0}+\frac{e_{a} v_{\|} E_{\|}}{T_{a}} f_{a 0},
$$

which was done by Hinton and Rosenbluth for the case of a large-aspect-ratio tokamak with circular cross section, where $B \simeq B_{0}(1-\epsilon \cos \theta)$. It appears difficult generalize their calculation to arbitrary stellarator geometry, but it is possible to extend it to a tokamak with arbitrary aspect ratio and shape of the flux surfaces. As we shall see, the result then also applies to an important class of stellarators.

If we write $\mathbf{B}=I(\psi) \nabla \varphi+\iota \nabla \varphi \times \nabla \psi$, where $\psi$ is the toroidal flux, $\iota=q^{-1}$ the rotational transform, and $I(\psi)=R B_{\varphi}$, then the radial excursion (in terms of $\psi$ ) of a particle orbit is given by

$$
\Delta_{a}=\frac{I v_{\|}}{\iota \Omega_{a}}
$$

Thus $\mathbf{v}_{d} \cdot \nabla \psi=v_{\|} \nabla_{\|} \Delta_{a}$ and the drift kinetic equation (15) can be written as

$$
v_{\|} \nabla_{\|} g_{a}-C_{a}\left(g_{a}+F_{a}\right)=\frac{e_{a} v_{\|} E_{\|}}{T_{a}} f_{a 0},
$$

where $g_{a}=f_{a 1}-F_{a}$ and

$$
F_{a}=-\Delta_{a} \frac{\partial f_{a 0}}{\partial \psi} .
$$

Expanding $g_{a}=g_{a 0}+g_{a 1}+\cdots$ as usual in the smallness of the collisionality and using pure pitch-angle scattering in the collsion operator yields the conventional solution

$$
\begin{aligned}
g_{a 0} & =\frac{\sigma s_{a} v}{2 \nu_{D a}} \int_{\lambda}^{1 / B_{\max }} \frac{d \lambda^{\prime}}{\left\langle\sqrt{1-\lambda^{\prime} B}\right\rangle}, \\
s_{a} & =\frac{e_{a}\left\langle E_{\|} B\right\rangle}{T_{a}} f_{a 0}+\frac{\nu_{D a} I B}{\iota \Omega_{a}} \frac{\partial f_{a 0}}{\partial \psi},
\end{aligned}
$$

in analogy with Eq. (11).

As already mentioned, the collision operator must be retained in the boundary layer, where however the source terms in the kinetic equation (17) are unimportant, so we need to solve the equation

$$
\mathbf{B} \cdot \nabla g_{a}=\frac{2 \nu_{D}^{a}}{v} \frac{\partial}{\partial \lambda}\left(\lambda \xi \frac{\partial g_{a}}{\partial \lambda}\right) .
$$


In this equation, it is permissible to approximate $\lambda$ by $1 / B_{\max }$, and the solution should match the $\lambda$-derivative of Eq. (19),

$$
\frac{\partial g_{a}}{\partial \lambda} \rightarrow-\frac{\sigma v s_{a}}{2 \nu_{D}^{a}\langle\sqrt{1-\lambda B}\rangle} .
$$

away from the boundary layer on the circulating side.

In most of the layer, except in a sublayer close to the turning points, the term $\partial^{2} g_{a} / \partial \lambda^{2}$ is dominant and we have

$$
\nabla_{\|} g_{a}=\frac{2 \sigma \nu_{D}^{a}}{v b B_{\max }^{2}} \sqrt{1-b} \frac{\partial^{2} g_{a}}{\partial \lambda^{2}}
$$

where $b=B / B_{\max }$. It is now convenient to introduce new independent variables by defining

$$
\begin{gathered}
x(\lambda)=\frac{\lambda B_{\max }-1}{\sqrt{\nu_{a 0}}}, \\
y(l)=\sigma \pi \int_{l_{\max }}^{l} \sqrt{1-b\left(l^{\prime}\right)} \frac{d l^{\prime}}{b\left(l^{\prime}\right)} / \oint \sqrt{1-b\left(l^{\prime}\right)} \frac{d l^{\prime}}{b\left(l^{\prime}\right)},
\end{gathered}
$$

where $d l=d \theta / \nabla_{\|} \theta$ is the arc length along the field as in the previous section, and

$$
\nu_{a 0}=\frac{2 \nu_{D}^{a}}{\pi v} \oint \sqrt{1-b(l)} \frac{d l}{b(l)} .
$$

In the new variables, Eq. (22) reduces to the ordinary diffusion equation

$$
\frac{\partial g_{a}}{\partial y}=\frac{\partial^{2} g_{a}}{\partial x^{2}}
$$

in the domain $-\pi<y<\pi,-\infty<x<\infty$, see Fig 1 . The boundary conditions are, however, unorthodox and make the equation non-trival to solve. The region $x<0$ corresponds to circulating particles and $x>0$ to trapped ones. Continuity at the bounce points requires

$$
\begin{array}{ll}
g_{a}(x, 0-)=g_{a}(x, 0+), & x>0 \\
g_{a}(x,-\pi)=g_{a}(x, \pi), & x>0
\end{array}
$$

whilst periodicity in the counter-passing $(y<0)$ and co-passing $(y>0)$ domains implies

$$
\begin{array}{ll}
g_{a}(x,-\pi)=g_{a}(x, 0-), & x<0 \\
g_{a}(x, \pi)=g_{a}(x, 0+), & x<0
\end{array}
$$


but $g_{a}$ need not be continuous at $y=0$ for negative $x$. The point is that pitchangle scattering causes diffusion in the $x$-direction between the co- and counter-passing domains on the one hand and the trapped region on the other, but there is no direct exchange between the co- and counter-passing regions, see Fig. 1.

Remarkably, this boundary-value problem is identical to one arising in the theory of transport across magnetic islands [18]. The solution, which can be obtained with the Wiener-Hopf method, has the asymptotic behavior

$$
g_{a}(x, y) \rightarrow 0, \quad x \rightarrow+\infty
$$

on the trapped side of the boundary layer and

$$
g_{a}(x, y) \rightarrow \sigma\left(c_{0}+c_{1} x\right), \quad x \rightarrow-\infty
$$

on the circulating side. Here $\sigma=-y /|y|$,

$$
\frac{c_{0}}{c_{1}}=\sqrt{2}(\sqrt{2}-1) \zeta\left(\frac{1}{2}\right) \simeq-0.855
$$

and $\zeta$ denotes the Riemann zeta function. Matching to the collisionless solution (21) in the circulating region determines

$$
c_{1}=-\frac{s_{a} v \sqrt{\nu_{a 0}}}{2 \nu_{D}^{a} B_{\max }\langle\sqrt{1-b}\rangle}
$$

and thus

$$
g_{a}=\text { Eq. }(19)+0.855 c_{1} \sigma .
$$

Having thus solved the boundary layer problem, we proceed to calculate the current from the two terms in $s_{a}$, Eq. (20). The first term produces a current proportional to an effective conductivity

$$
\left\langle J_{a \|} B\right\rangle=\sigma_{\text {eff }}\left\langle E_{\|} B\right\rangle,
$$

which is smaller than the Spitzer value (for the scattering collision operator we use) because of the existence of trapped particles. Evaluating the current

$$
\left\langle J_{a \|} B\right\rangle=\left\langle e_{a} B \int v_{\|} g_{a} d^{3} v\right\rangle
$$

using Eq. (26) gives

$$
\sigma_{\mathrm{eff}}=\frac{n_{a} e_{a}^{2}}{m_{a}}\left\{\frac{f_{c}^{\mathrm{eff}}(v)}{\nu_{D a}}\right\},
$$


where the brackets denote a velocity-space average [8]

$$
\{\cdots\}=\int_{0}^{\infty}(\cdots) \frac{m_{a} v^{2}}{3 T_{a}} \frac{f_{a 0}}{n_{a}} 4 \pi v^{2} d v,
$$

and

$$
f_{c}^{\mathrm{eff}}=f_{c}+\delta f_{c}(v)
$$

is an effective fraction of circulating particles [14]. The collisionless value (obtained from taking $\left.g_{a}=(19)\right)$ is

$$
f_{c}=\frac{3\left\langle B^{2}\right\rangle}{4} \int_{0}^{1 / B_{\max }} \frac{\lambda d \lambda}{\langle\sqrt{1-\lambda B}\rangle},
$$

and the correction from the boundary layer is

$$
\delta f_{c}=\frac{3 \cdot 0.855}{4} \cdot \frac{\left\langle b^{2}\right\rangle}{\langle\sqrt{1-b}\rangle} \sqrt{\nu_{a 0}}=0.51\left\langle b^{2}\right\rangle \sqrt{\frac{\nu_{D}^{a}}{v\langle\sqrt{1-b}\rangle} \oint \frac{d l}{b}}
$$

In a large-aspect-ratio tokamak with circular cross section, $B=B_{0}(1-\epsilon \cos \theta)$, we find

$$
\begin{gathered}
\langle\sqrt{1-b}\rangle \simeq \frac{2 \sqrt{2 \epsilon}}{\pi}, \\
\oint \frac{d l}{b} \simeq \frac{2 \pi R}{\iota}
\end{gathered}
$$

and hence

$$
f_{c}^{\mathrm{eff}} \simeq 1-1.46 \epsilon^{1 / 2}+\frac{1.35 \nu^{* 1 / 2}}{\epsilon^{1 / 4}},
$$

where $\nu^{*}=\nu_{D}^{a} R / \iota v$. These relations show how the "effective" fraction of trapped particles $\left(1.46 \epsilon^{1 / 2}\right.$ in a standard tokamak) is reduced by a factor proportional to the square root of the collisionality, which thus increases the parallel conductivity.

We now turn to the bootstrap current, which is produced by the second term in Eq. (20). Evaluating the current in this case gives

$$
\begin{gathered}
\left\langle J_{a \|} B\right\rangle=\left\langle e_{a} B \int v_{\|}\left(F_{a}+g_{a}\right) d^{3} v\right\rangle \\
=-\frac{I p_{a}}{\iota}\left\{\left(1-f_{c}^{\mathrm{eff}}(v)\right)\left[A_{1 a}+\left(\frac{m_{a} v^{2}}{2 T_{a}}-\frac{5}{2}\right) A_{2 a}\right]\right\},
\end{gathered}
$$

where

$$
\begin{gathered}
A_{1 a}=\frac{d \ln p_{a}}{d \psi}+\frac{e_{a}}{T_{a}} \frac{d \Phi}{d \psi}, \\
A_{2 a}=\frac{d \ln T_{a}}{d \psi} .
\end{gathered}
$$

The reduction in the effective number of trapped particles due to a small but finite collisionality thus leads to a reduced bootstrap current. 


\section{B. Quasi-isodynamic stellarator}

The calculation above applies to an axisymmetric tokamak and appears difficult to generalize to general stellarator geometry. Of course, it immediately applies to quasiaxisymmetric and quasihelically symmetric configurations, since their neoclassical properties are identical (in leading order) to those in tokamaks $[19,20]$. However, there is another important but less trivial case amenable to a similar analysis, and this is the case of a perfectly quasi-isodynamic stellarator [21], i.e., a stellarator where the contours of constant $|\mathbf{B}|$ are poloidally closed and the bounce-averaged radial drift vanishes for all particle orbits,

$$
\int \frac{\left(\mathbf{v}_{d a} \cdot \nabla r\right) d l}{v_{\|}}=0
$$

where the integral is taken between two successive bounce points. The latter property is also referred to as omnigeneity in the literature [22]. In such a magnetic field, it can be shown that the minimum and maximum magnetic field strength, $B_{\min }$ and $B_{\max }$, are the same for all field lines on the same flux surface [23]. This circumstance makes it convenient to use $(r, \alpha, B)$ as real-space coordinates, where $\alpha=\theta-\iota \varphi$ in Boozer coordinates. The first two coordinates then label the field line, and the position along it is indicated by the magnetic field strength $B$. (In general, there will be an even number of points with the same field strength in one period, so an additional discrete variable is required for distinguishing these from one another.) Furthermore, it can be shown that every integral of the form

$$
\int_{l_{1}}^{l_{2}} F(B) d l
$$

taken along a field line between points of equal field strength (bounce points), is independent of the field line label $\alpha$ and only depends on their flux surface $r$ [23].

A further property of quasi-isodynamic fields is that it is possible describe the orbits in a simple way. If we write $\mathbf{B}=\nabla \psi \times \nabla \alpha$, then the radial excursion (in terms of $\psi$ ) for passing particle orbits can be written as [21]

$$
\Delta_{a}=-\frac{\mu_{0} J(\psi)}{2 \pi} \frac{v_{\|}}{\Omega_{a}}+\frac{\partial}{\partial \alpha} \int_{B}^{B_{\max }} h\left(\psi, \alpha, B^{\prime}\right) \frac{\partial}{\partial B^{\prime}}\left(\frac{\sigma m_{a} v \sqrt{1-\lambda B^{\prime}}}{e_{a} B^{\prime}}\right) d B^{\prime}, \quad \lambda<1 / B_{\max },
$$

where $J(\psi)$ is the toroidal current enclosed by the flux surface $\psi$, and the function $h$ encapsulates all other necessary geometric information about the magnetic field. Note 
the similarity between Eq.(16) and the first term in Eq. (34). As shown in Ref. [21], it then follows that the first-order distribution function is equal to

$$
f_{a 1}=F_{a}+g_{a 0}
$$

with $F_{a}=(18)$ as in the tokamak case, and as a result of the $\alpha$-derivative in Eq. (34), which disappears on an orbit average, the equation for $g_{a 0}$ is also the same as in a tokamak, with the replacement $I(\psi) \rightarrow-\iota \mu_{0} J(\psi) / 2 \pi$. The solution therefore coincides with Eq. (19), see Ref. [21] and [24] for details. In particular, if the flux surface in question does not enclose any net toroidal current, $J(\psi)=0$, which is the usual situation in a stellarator, then $g_{a 0}=0$ and the bootstrap current vanishes, since $F_{a}$ does not carry any net current.

Turning to the collisional correction to the bootstrap current, we note that the fact that $\partial B_{\max } / \partial \alpha=0$ makes it possible to reduce the kinetic equation (22) for the boundary layer to the diffusion equation (24) in each one of the magnetic wells along a field line. In contrast to the tokamak, these wells are not identical since $B$ depends on the arc length differently in each well. But because of the property (33), the quantity $\nu_{a 0}=(23)$ is the same for each well. This implies that the matching condition (25) is also well-independent, and that the distribution function away from the boudary layer is given by Eq. (26). The collisional correction to the bootstrap current is thus the same as in a tokamak with the replacement $I(\psi)=-\iota \mu_{0} J(\psi) / 2 \pi$ and vanishes on flux surfaces where $J(\psi)=0$. Thus we conclude that the bootstrap current in a quasiisodynamic stellarator vanishes to a very high degree of accuracy: not only is there no current in the collisionless limit (as known from Ref. [21]), but the leading correction due to finite collisionality is also absent.

\section{Comparison with numerical results}

We now try to numerically verify some of the analytical predictions that have been made. The first step is the construction of a suitable magnetic equiblibrium. In order to display the effects of finite aspect ratio on the bootstrap current in a tokamak, we choose a high-beta-poloidal discharge of the National Spherical Torus Experiment (NSTX, discharge 133964 at $t=505 \mathrm{~ms}$ ) described in Ref. [25]. The plasma current was $0.7 \mathrm{MA}$, the toroidal field on axis $0.48 \mathrm{~T}$, and the edge safety factor $q_{95}=14$. 
The magnetic equilibrium was calculated with the VMEC code [26] in fixed-boundary mode with specified pressure and rotation transform profiles and with the boundary taken at the normalized poloidal flux surface $\psi_{N}=0.9875$. The beta value was found to be $42.4 \%$ on axis and $8.3 \%$ on a volume average. The use of Boozer coordinates proved arduous because of the strong variation of the Jacbian $\sim 1 / B^{2}$ over the outer flux surfaces, making it necessary to use hundreds of Fourier modes to attain sufficient accuracy near the edge.

The neoclassical calculations were performed with the DKES (Drift Kinetic Equation Solver) code [27, 28], which uses a Fourier-Legendre representation of the distribution function to solve the drift kinetic equation by a variational principle. At very low collisionalities, the first-order distribution function becomes highly localized in phase space and thus requires a large number of Fourier-Legendre modes. In particular for stellarators with complex Fourier spectra, it is in general impossible to numerically verify the collisionless limit for the bootstrap current coefficient given in Eq. (14) [29]. For symmetric configurations, however, this problem is much less severe. In the present NSTX example, it was nevertheless necessary to use up to 500 Fourier modes and 400 Legendre polynomials for the distribution function. The $B_{m 0}$-Fourier spectrum (with $m$ being the poloidal mode number) in Boozer coordinates becomes rather broad near the edge. Here, the analysis is limited to about $85 \%$ of the plasma radius and up to $100 B_{m 0}$-Fourier modes are taken into account.

The effective circulating-particle fraction $f_{c}^{\text {eff }}$ defined by Eqs. (27) and (28) can be obtained from the parallel mono-energetic transport coefficient $D_{33}$ calculated by DKES [14]. For low collisionalities, the result was fitted to the ansatz $f_{c}^{\text {eff }}\left(\nu^{*}\right)=$ $\alpha_{0}+\alpha_{1} \sqrt{\nu^{*}}$ by a least-squares technique which includes the uncertainties of the $D_{33}$ transport coefficient at low collisionalities in the weighting scheme. As found in the previous section, $\alpha_{1} \sqrt{\nu^{*}}$ describes the contribution of the boundary layer to the parallel conductivity. With decreasing $\nu^{*}$, the uncertainty in $D_{33}$ increases whereas at higher $\nu^{*}$ a systematic deviation from the fit appears before the transition to the plateau regime. An extendend plateau regime exists at the innermost radii, which reduces the $\nu^{*}$-range for the fitting.

Figure 2 shows the results of the fitting of the DKES data and the analytical expression of the boundary layer contribution given in Eq. (30) as well as the large- 
aspect-ratio-tokamak limit. The results are displayed as functions of the minor radius represented by the local inverse aspect ratio, $\epsilon=\psi^{\prime} / I$, where $\psi^{\prime}$ is the radial derivative of the toroidal flux. Note that according to this standard stellarator definition, $\epsilon$ exceeds unity in the edge region of NSTX. For all $\epsilon$, the fitting coefficients $\alpha_{0}$ and $\alpha_{1}$ are in very good agreement with the circulating particle fraction (29) and the analytical result (30) from the boundary layer analysis, respectively. We can thus conclude that Eqs. (28)-(30) provide an accurate description of the parallel transport coefficient at low collisionalities.

We have already noted in Eq. (32) that the transport coefficient for the bootstrap current is essentially the same as that for the parallel conductivity (28). This is a general property of axisymmetric configurations, where the parallel friction and the radials transport are related by the expression

$$
\mathbf{B} \cdot \nabla B=-\iota \epsilon(\mathbf{B} \times \nabla B) \cdot \nabla r
$$

which leads to a link between the mono-energetic transport coefficients [29, 30]. For other types of symmetry, a similar relationship exists. In symmetric devices (in contrast to a general stellarator), from a single mono-energetic transport coefficient, the other coefficients can thus be computed. In DKES notation, the bootstrap current coefficient, $D_{31}$, is given by

$$
D_{31}=\frac{2}{3 \iota \epsilon}\left(1-f_{c}^{\mathrm{eff}}\right) .
$$

Figure 3 shows this coefficient computed numerically as well as the result obtained from the analytic representation (30) of $f_{c}^{\text {eff }}$ and the large-aspect-ratio limit given by the last term in Eq. (31) but using the exact $f_{c}$ value (29) instead of the first two terms. The magnetic geometry is the same as in Fig. 2, and the calculation is done at three different radii. The DKES results at low collisionality again confirm the boundary-layer analysis. With increasing $\epsilon$, the plateau regime shrinks and is shifted to higher $\nu^{*}$. The difference bewteen the full analaltical result (solid lines) and the large-aspect-ratio limit (dashed lines) increases with $\epsilon$.

\section{The bootstrap current in a general stellarator}

As already remarked, the low-collisionality limit (14) is valid in general stellarator geometry, but the finite-collisionality correction calculated in Sec. V is restricted to 
axisymmetric, quasisymmetric and quasi-isodynamic devices. A qualitative difference between these configurations and the general stellarator is that the maximum field strength on each flux surface is attained along a curve, and not in a point, like in a general stellarator. This circumstance could affect the structure of the collisional boundary layer between trapped and circulating particles, and makes an analytical treatment difficult.

In order to explore the effect of finite collisionality numerically instead, we use the DKES code to analyze the behavior of the $D_{31}$ bootstrap current coefficient. A classical stellarator configuration is best suited for this analysis, since the number of Fourier harmonics of the field strength is then kept low, making it possible to achieve relatively good numerical accuracy at low collisionality. The standard configuration of the Large Helical Device (LHD) with $R=3.75$ at half the plasma radius was chosen for the task, with the side-band $B_{m n}$-Fourier modes truncated, so that the only components are $B_{00}=1, B_{1,0}=-0.07271$ and $B_{21}=0.05063$. Figure 4 shows the bootstrap current coefficient normalized to the collisionless asymptotic value of a circular tokamak in the large-aspect-ratio limit as function of collisionality and radial electric field. The latter has no effect on the bootstrap current in a tokamak or quasisymmetric stellarator in leading order; orbit-squeezing effects are beyond the standard neoclassical ordering adopted here. The electric field is here treated in the conventional "mono-energetic" approximation of DKES described in Refs. [27, 28, 29]. This treatment is not rigorous and cannot be trusted when the parameter $E_{r} / v B$ approaches $\iota \epsilon[29,31]$. Therefore, this parameter is kept very small in the present simulations. At very low $\nu^{*}$, the DKES $D_{31}$ values have a rather low accuracy indicated by the error bars, in particular for small $E_{r} / v B$. Nevertheless, a weighted least-squares fit to an expression $D_{31}=\beta_{0}+\beta_{1} \sqrt{\nu^{*}}$ similar to that used above 3 has been performed and is represented by the solid lines in the figure. The approach to the collisionless Shaing-Callen asymptote (14) is seen to depend on the radial electric field, and is slowest when $E_{r}=0$. Except when the electric field is relatively large, $\beta_{0}$ agrees well with Eq. (14), and it appears that the collisional correction scales as $\sqrt{\nu^{*}}$, like in the tokamak case. 


\section{Conclusions}

Several results concerning the bootstrap current in stellarators and tokamak are reported in the present paper. First, the expression (14) for the bootstrap current originally derived by Shaing and Callen has been recovered by a method that does not rely on the Hirshman-Sigmar moment formalism and makes no distinction between tokamak and stellarator geometry. This expression applies to arbitrary magnetic geometry with nested flux surfaces in the long-mean-free-path limit. Second, the correction due to a small but finite collisionality has been considered, following Hinton and Rosenbluth, whose calculation has been generalized to arbitrary axisymmetric geometry. This correction is proportional to the square root of the collisionality and tends therefore to be important in practice. In fact, in most stellarators the long-mean-free-path limit is approached so slowly at small collisionality that the correction is predicted to increase or decrease the bootstrap current significantly in most relevant plasma scenarios [29]. Third, it has been shown that although the finite-collisionality correction does not apply to a general stellarator, it is applicable to quasi-isodynamic configurations. In particular, if the net toroidal current in the plasma volume inside a flux surface vanishes, then so does the bootstrap current (including the finite-collisionality correction) on that surface. This conclusion strengthens the result found in Ref. [21] that the bootstrap current automatically becomes negligible in a sufficiently well optimized stellarator of the quasi-isodynamic type. Finally, the drift kinetic equation has been solved numerically using the DKES code, and the dependence on the collisionality has been found to agree very well with the analytical result in spherical tokamak geometry. In a classical stellarator, the finite-collisionality correction to the bootstrap current appears to have scaling similar to that in the tokamak, with a coefficient that depends on the radial electric field.

\section{Acknowledgment}

It is a pleasure to thank Stefan Gerhardt for providing NSTX equilibrium data, John Canik for helping to set up the VMEC runs, and Jürgen Nührenberg for originally suggesting that the first collisionality correction to the bootstrap current should vanish in a quasi-isodynamic stellarator. 


\section{References}

[1] A.A. Galeev, Sov. Phys. JETP 32, 752 (1971).

[2] R. Bickerton, J.W. Connor and J.B. Taylor, Nature Phys. Sci. 229, 110 (1971).

[3] M.C. Zarnstorff and S. Prager, Phys. Rev. Lett. 53, 454 (1984); Phys. Fluids 29, 298 (1986).

[4] K.C. Shaing and J.D. Callen, Phys. Fluids 26, 3315 (1983).

[5] A.H. Boozer and H.J. Gardner, Phys. Fluids B2, 2408 (1990).

[6] V.V. Nemov, S.V. Kasilov, W. Kernbichler and M.F. Heyn, Phys. Plasmas 6, 4622 (1999).

[7] W. Kernbichler, V.V. Nemov, S.V. Kasilov and M.F. Heyn, Problems of Atomic Science and Technology. Series: Plasma Physics (6), 8 (2000).

[8] P. Helander and D.J. Sigmar, Collisional transport in magnetized plasmas (Cambridge University Press, 2002).

[9] T.M. Antonsen and M.S. Chu, Phys. Fluids 25, 1295 (1982).

[10] M.N. Rosenbluth, R.D. Hazeltine, and F.L. Hinton, Phys Fluids 15, 116 (1972).

[11] M. Taguchi, Plasma Phys. Contr. Fusion 30, 1897 (1988).

[12] H. Maaßberg and C. D. Beidler, Phys. Plasmas 17, 052507 (2010).

[13] H. Sugama and S. Nishimura, Phys. Plasmas 9, 4637 (2002); 15, 042502 (2008).

[14] H. Maaßberg, C. D. Beidler, and Y. Turkin, Phys. Plasmas 16, 072504 (2009).

[15] N. Nakajima, M. Okamoto, J. Todoroki, Y. Nakamura and M. Wakatani, Nucl. Fusion 29, 605 (1989).

[16] S.P. Hirshman and D.J. Sigmar, Nucl. Fusion 21, 1079 (1981).

[17] F.L. Hinton and M.N. Rosenbluth, Phys. Fluids 16, 836 (1973).

[18] R.D. Hazeltine, P. Helander, and P.J. Catto, Phys. Plasmas 4, 2920 (1997). 
[19] A.H. Boozer, Phys. Fluids 26, 496 (1983).

[20] P. Helander and A.N. Simakov, Phys. Rev. Lett. 101, 145003 (2008).

[21] P. Helander and J. Nührenberg, Plasma Phys. Control. Fusion 51, 055004 (2009).

[22] L.S. Hall and B. McNamara, Phys. Fluids 18, 552 (1975).

[23] J.R. Cary and S. Shasharina, Phys. Plasmas 4, 3323 (1997).

[24] M. Landreman and P.J. Catto, Plasma Phys. Control. Fusion 53035016 (2011).

[25] S.P. Gerhardt, E. Fredrickson, D. Gates, S. Kaye, J. Menard, M.G. Bell, R.E. Bell, B.P. Le Blanc, H. Kugel, S.A. Sabbagh and H. Yuh, Nucl. Fusion 51, 033004 (2011).

[26] S.P. Hirshman and J.C. Whitson, Phys. Fluids 26, 3553 (1983).

[27] S.P. Hirshman, K.C. Shaing, W.I. van Rij, C.O. Beasley, and E.C. Crume, Phys. Fluids 29, 2951 (1986).

[28] W.I. van Rij and S.P. Hirshman, Phys. Fluids B1, 563 (1989).

[29] C.D. Beidler, K. Allmaier, M.Yu. Isaev, S.V. Kasilov, W. Kernbichler, G.O. Leitold, M. Maaßberg, D.R. Mikkelsen, S. Murakami, M. Schmidt, D.A. Spong, V. Tribaldos and A. Wakasa, Nucl. Fusion 51, 076001 (2011).

[30] M. Taguchi, Phys. Fluids B4, 3638 (1992).

[31] M. Landreman, Plasma Phys. Contr. Fusion 53, 082003 (2011). 


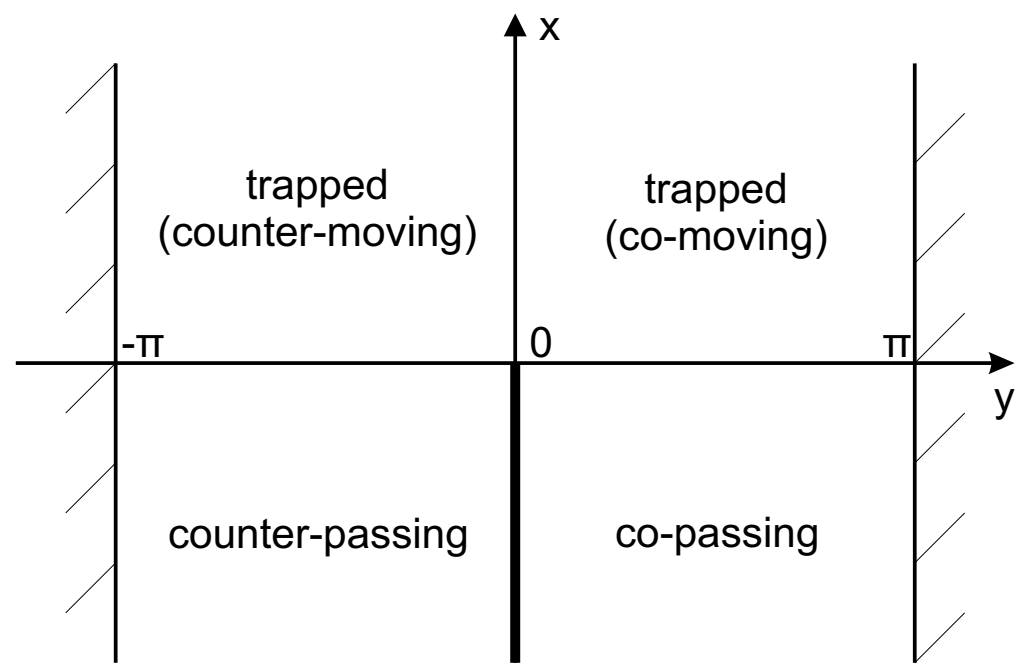

Figure 1: Domain representing the trapped-passing boundary layer, in which the diffusion equation is solved. 


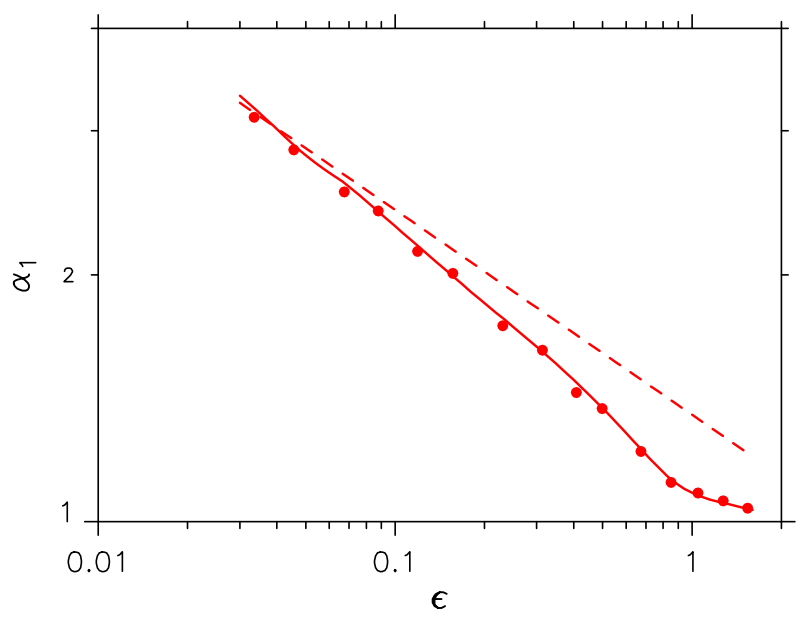

Figure 2: (color online) The boundary layer contribution coefficient, $\alpha_{1}$, vs. the local inverse aspect ratio, $\epsilon$ : fits to DKES results (full circle), analytical contribution from Eq. (30) (solid line), and the limit of a large-aspect-ratio tokamak with circular cross section (dashed line). 


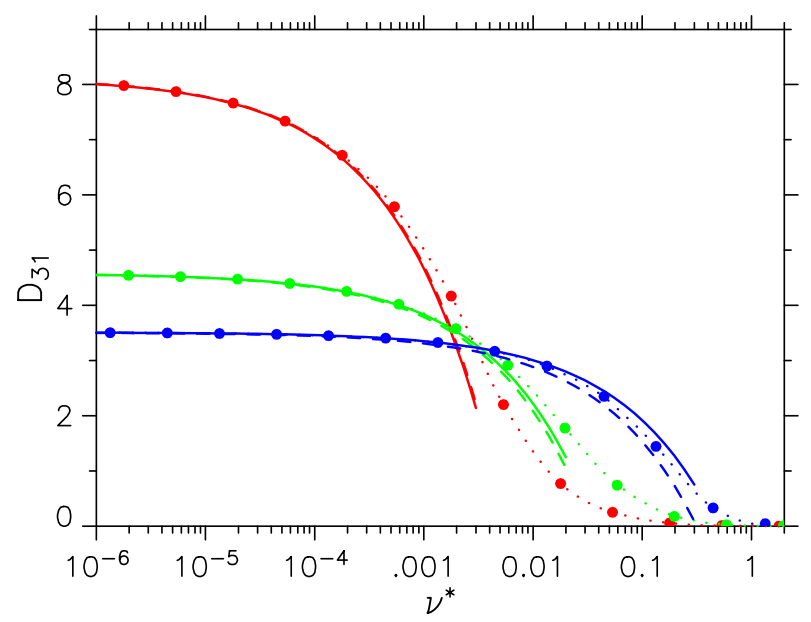

Figure 3: (color online) The mono-energetic bootstrap current coefficient (35) vs. collisionality, $\nu^{*}$, for $\epsilon=0.0335,0.119$ and 1.047 (from top to bottom at low $\nu^{*}$ ) with DKES results (full circles), the analytical form of $f_{c}^{\text {eff }}$ from Eqs. (29)-(30) (solid lines) and the limit of a large-aspect-ratio tokamak with circular cross section for $\delta f_{c}$ (dashed lines). (The dotted lines depict interpolations to the DKES data.) 


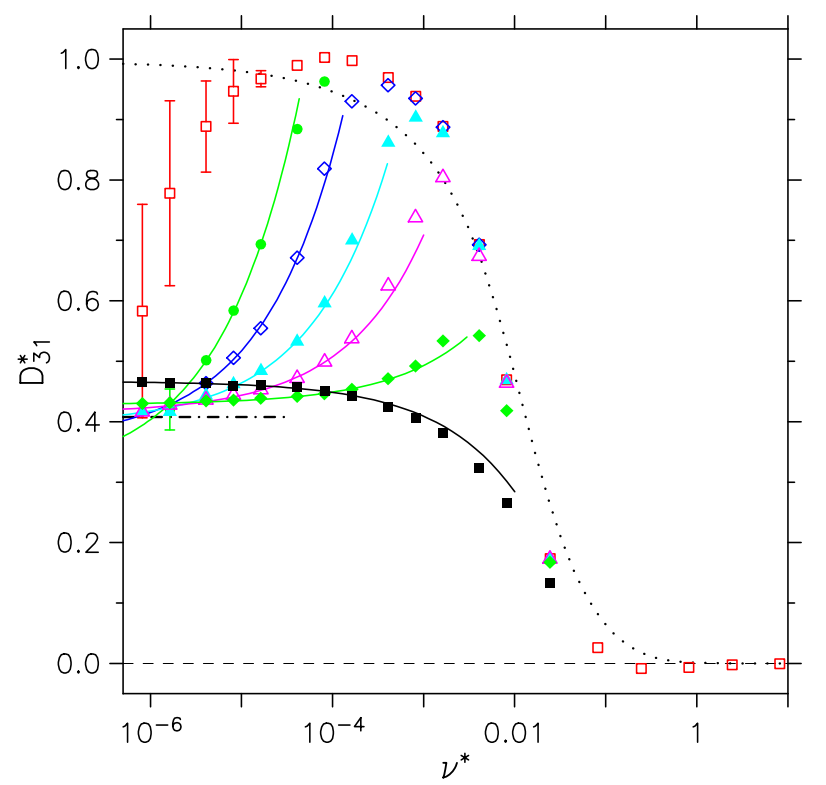

Figure 4: (color online) Normalized mono-energetic bootstrap current coefficient vs. collisionality, $\nu^{*}$, for different radial electric fields: $E_{r} / v B=0$ (open squares), $10^{-5}$ (full circles), $3 \cdot 10^{-5}$ (open diamonds), $10^{-4}$ (full triangles), $3 \cdot 10^{-4}$ (open triangles), $10^{-3}$ (full diamonds), $3 \cdot 10^{-3}$ (full squares). Solid lines are the fits to the DKES data at the lowest $\nu^{*}$. The Shaing-Callen value is indicated by the dot-dashed line, and the equivalent tokamak values by the dotted line. 